\title{
Mitochondrial dysfunction, a probable cause of persistent oxidative stress after exposure to ionizing radiation
}

\author{
Takako Yoshida, Shinji Goto, Miho Kawakatsu, Yoshishige Urata and Tao-Sheng Li \\ Department of Stem Cell Biology, Atomic Bomb Disease Institute, Nagasaki University \\ Graduate School of Biomedical Sciences, Nagasaki 852-8523, Japan
}

Running title: Radiation-induced mitochondrial dysfunction

Address correspondence to: Shinji Goto, Ph.D., Department of Stem Cell Biology, Atomic Bomb Disease Institute, Nagasaki University Graduate School of Biomedical Sciences, Nagasaki 852-8523, Japan

Tel. +81-95-819-7099; FAX. +81-95-819-7100

E-mail; sgoto@nagasaki-u.ac.jp

Abbreviations: ROS, reactive oxygen species; 8-OHdG, 8-hydroxy-2'-deoxyguanosine; ETC, electron transport chain; ${ }^{\circ} \mathrm{OH}$, hydroxyl radical; $\mathrm{H}_{2}$ DCFDA, 2',7'-dichlorodihydrofluorescein diacetate

Keywords: radiation, reactive oxygen species, mitochondrial dysfunction, oxidative stress, 8-hydroxy-2'-deoxyguanosine

\begin{abstract}
Several recent studies have suggested that the reactive oxygen species (ROS) generated from mitochondria contribute to genomic instability after exposure of the cells to ionizing radiation, but the mechanism of this process is not yet fully understood. We examined the hypothesis that irradiation induces mitochondrial dysfunction to cause persistent oxidative stress, which contributes to genomic instability. After the exposure of cells to 5 Gy gamma-ray irradiation, we found that the irradiation induced the following changes in a clear pattern of time courses. First, a robust increase of intracellular ROS levels occurred within minutes, but the intracellular ROS disappeared within 30 minutes. Then the mitochondrial dysfunction was detected at 12 hours after irradiation, as indicated by the decreased activity of NADH dehydrogenase (Complex I), the most important enzyme in regulating the release of ROS from the mitochondrial electron transport chain (ETC). Finally, a significant increase of ROS levels in the mitochondria and the oxidation of mitochondrial DNA were observed in cells at 24 hours or later after irradiation. Although further experiments are required, results in this study support the hypothesis that mitochondrial dysfunction causes persistent oxidative stress that may contribute to promote radiation-induced genomic instability.
\end{abstract}




\section{Introduction}

It is well known that ionizing radiation, such as gamma-rays, induces DNA double-strand breaks (DSBs). In mammalian cells, the failure to repair DSBs leads to chromosomal rearrangements, cell cycle arrest, or cell death [1]. However, a high-frequency of chromosomal deletions, mutations, translocations, and micronuclei have been observed in the progeny of irradiated cells long after the initial radiation exposure. These phenomena are referred to as radiation-induced genomic instabilities, which are thought to promote carcinogenesis [2]. Although several studies have recently suggested a relationship between the radiation-induced genomic instability and the reactive oxygen species (ROS) generated by the mitochondria [3-7], it is poorly understood how irradiation induces the generation of ROS by the mitochondria.

Mitochondria are the primary energy-generating organelles in most eukaryotic cells. Mitochondria synthesize ATP by oxidative phosphorylation in the electron transport chain (ETC). During this process, the superoxide anion $\left(\mathrm{O}_{2}^{-}\right)$is generated due to a one-electron reduction of molecular oxygen $\left(\mathrm{O}_{2}\right)$ by the electron leaked from the ETC [8]. Inhibiting the activity of the mitochondrial ETC complexes enhances the leakage of electrons and the subsequent generation of $\mathrm{O}_{2}^{-}[9-12]$.

However, ionizing radiation is known to ionize water molecules $\left(\mathrm{H}_{2} \mathrm{O}\right)$ and result primarily in the generation of the hydroxyl radical $\left({ }^{\circ} \mathrm{OH}\right)$, the most toxic form of ROS $[8,13]$. These ${ }^{\circ} \mathrm{OH}$ have the ability to oxidize biomolecules, including proteins or lipids $[13,14]$. The mitochondrial inner membrane contains phospholipids, such as phosphatidylcholine, phosphatidylethanolamine, and cardiolipin, which play very important roles in the optimal functioning of numerous enzymes involved in the ETC of mitochondria $[15,16]$. Alterations in the membrane lipid profile, such as a decrease in lipid content, peroxidation, and changes in composition may result in or enhance the generation of $\mathrm{O}_{2}{ }^{-}$arising from leakage of electrons from ETC component enzymes. Therefore, ${ }^{\circ} \mathrm{OH}$ generated by irradiation may induce oxidative damage of the mitochondria through peroxidation of the above-mentioned phospholipids and 
then enhance the generation of $\mathrm{O}_{2}{ }^{-}$by the mitochondria.

In this study, we examined whether exposure to ionizing radiation induces mitochondrial dysfunction, and then we investigated how the mitochondrial dysfunction leads to persistent oxidative stress that may contribute to promote genomic instability after irradiation.

\section{Materials and methods}

Materials

Dulbecco's modified Eagle's medium (DMEM), the DNA Extractor TIS Kit, and the mtDNA Extractor CT Kit were purchased from Wako Pure Chemical Industries, Ltd. (Osaka, Japan). Fetal bovine serum (FBS), 2',7'-dichlorodihydrofluorescein diacetate ( $\left.\mathrm{H}_{2} \mathrm{DCFDA}\right)$,

Hanks' Balanced Salt Solution (HBSS), and MitoSOX ${ }^{\mathrm{TM}}$ Red mitochondrial superoxide indicator were purchased from Invitrogen Corp. (Carlsbad, CA). The BCA Protein Assay Reagent Kit was purchased from Thermo Fisher Scientific Inc. (Waltham, MA). The 8-hydroxy-2'-deoxyguanosine (8-OHdG) Check ELISA kit was purchased from the Japan Institute for the Control of Aging, Nikken SEIL Co., Ltd. (Shizuoka, Japan). The other chemicals and reagents were purchased from Sigma Aldrich (St. Louis, MO).

\section{Cell culture and gamma-ray irradiation}

We used rat A7r5 cells for this study [17]. The cells were cultured in DMEM supplemented with $10 \% \mathrm{FBS}$ at $37^{\circ} \mathrm{C}$ under a humidified atmosphere of $5 \% \mathrm{CO}_{2}$. The cells at approximately $80 \%$ confluence were irradiated with a ${ }^{137} \mathrm{Cs}$ source at a dose rate of $1 \mathrm{~Gy} /$ minute up to 5 Gy with a PS-3100SB gamma-ray irradiation system (Pony Industry Co., Ltd. Osaka, Japan). The medium was replaced 1 hour before and 6 hours after the irradiation, and then it was replaced daily. To estimate how irradiation affects the growth of cells, we recorded the morphological changes with digital camera under microscopy at 24 and 72 hours after irradiation. The numbers of survived cells were counted by a cell counter of Nucleocounter 
(Chemotetec A/S, Denmark).

\section{Detection of intracellular ROS}

The intracellular ROS levels were measured based on the oxidation of $\mathrm{H}_{2}$ DCFDA to form the fluorescent compound 2',7'-dichlorofluorescein (DCF) [18-21]. Briefly, cells were cultured on 35 mm glass-base dishes (Asahi Glass Co., Ltd., Tokyo, Japan). Cells at approximately $80 \%$ confluence were incubated with $10 \mu \mathrm{M} \mathrm{H}_{2}$ DCFDA for 20 minutes before irradiation. The cells were irradiated and incubated at $37^{\circ} \mathrm{C}$ for $5,30,60$, or 120 minutes after irradiation. After the cells were washed with HBSS, the intracellular ROS were directly observed with a fluorescence microscope (IX-71, Olympus, Tokyo, Japan).

\section{Measurement of the activity of NADH dehydrogenase}

The activity of NADH dehydrogenase (mitochondrial ETC complex I), an enzyme located in the inner mitochondrial membrane that catalyzes the transfer of electrons from NADH to coenzyme Q, was measured as previously described, with a slight modification [22-24]. Briefly, the cells were detached with $0.05 \%$ trypsin and $0.5 \mathrm{mM}$ EDTA at $0,12,24,48$, or 72 hours after irradiation and washed twice with PBS containing protease inhibitors (1 mM phenylmethylsulfonylfluoride, $1 \mu \mathrm{g} / \mathrm{ml}$ leupeptin, $1 \mu \mathrm{g} / \mathrm{ml}$ pepstatin). The cells were resuspended in PBS with protease inhibitors and disrupted by a sonicator (BIORUPTOR UCD-200TM, Cosmo Bio, Tokyo, Japan) on ice. The homogenate was centrifuged at 12,500 x $g$ for 10 minutes, and the supernatant was used for measuring the activity of NADH dehydrogenase, the largest and most complicated enzyme of mitochondrial ETC. All measurements were performed in a $1-\mathrm{ml}$ quartz cuvette at $30^{\circ} \mathrm{C}$ using a UV-3100 PC spectrophotometer (Shimadzu, Kyoto, Japan). To compare the activities among samples collected at each time point, citrate synthase activity was also measured as described [25]. 


\section{Detection of ROS in mitochondria}

The level of ROS in the mitochondria was analyzed by flow cytometry with a MitoSOX Red mitochondrial superoxide indicator [26,27]. Briefly, the cells were detached with $0.05 \%$ trypsin and $0.5 \mathrm{mM}$ EDTA at $0,12,18,24$, or 72 hours after irradiation. The cells were resuspended in culture medium containing $5 \mu \mathrm{M}$ MitoSOX Red and incubated at $37^{\circ} \mathrm{C}$ for 10 minutes, avoiding exposure to light. After two washes with HBSS, the fluorescence intensity in the cells was estimated using a Cytomics FC 500 cytometer with FC500 CXP Cytometer software (Beckman coulter, Brea, CA).

\section{Evaluation of the oxidation of DNA in nuclei and mitochondria}

After $0,1,3,6,12,18,24,48$, or 72 hours of irradiation, the nuclear DNA and mitochondrial DNA were extracted from the cells with a DNA Extractor TIS Kit and an mtDNA Extractor CT Kit, respectively. The levels of 8-OHdG, one of the major biomarkers for the evaluation of oxidative DNA damage [28], were measured with an 8-OHdG Check ELISA kit according to the manufacturer's instructions.

\section{Statistical analysis}

The data are represented as the mean \pm standard error of the mean (SEM). The statistical significance was determined by the Student's $t$-test. Probability values less than 0.05 were considered significant. 


\section{Results}

Gamma-ray radiation inhibited the growth of cells without obvious morphological change

The most of the cells survived after irradiation, and the morphological findings in cells that survived the radiation were similar to those of non-radiated cells (Figure 1A). However, the total number of cells was significantly less 24 hours after irradiation when compared to that of the control group (Figure 1B). This indicated that irradiation significantly inhibited the growth of cells at a dose of $5 \mathrm{~Gy}$.

\section{Gamma-ray radiation rapidly induced an increase of intracellular ROS levels}

Although gamma-rays can directly ionize $\mathrm{H}_{2} \mathrm{O}$ to generate ${ }^{\circ} \mathrm{OH}$, the most toxic form of ROS, it is impossible to directly detect these ${ }^{\circ} \mathrm{OH}$ because of their very short life span $\left(10^{-9}\right.$ seconds) $[8,13]$. Alternatively, we monitored the intracellular ROS levels by fluorescence microscopy at 5, 30, 60, and 120 minutes after 5 Gy gamma-ray irradiation. As expected, a robust increase of intracellular ROS levels was detected after 5 minutes of irradiation (Figure 2). However, the intracellular ROS rapidly disappeared thereafter, and the intracellular ROS levels in these irradiated cells after 30 to 120 minutes were not obviously different from those of non-irradiated cells in which the intracellular ROS levels were barely detected at all time points during the 120 minute experiment. These results indicated that gamma-ray irradiation induced a robust, instantaneous increase of intracellular ROS levels within minutes.

\section{Gamma-ray radiation decreased the activity of NADH dehydrogenase}

It is generally accepted that oxidative stress persists over a long period of time after exposure to irradiation [29]. Considering that mitochondria are the principal generator of ROS [30-33], we speculated that irradiation might induce mitochondrial ETC dysfunction to perpetuate oxidative stress. To test this possibility, we measured the activity of NADH dehydrogenase (Complex I). We found that the activity of NADH dehydrogenase decreased to 
approximately $50 \%$ of the baseline value after 12 hours, and this lower activity persisted until 72 hours after irradiation (Figure 3). This finding suggests that mitochondrial dysfunction occurs within 12 hours after irradiation.

\section{Persistent increase of ROS levels in mitochondria after irradiation}

To further investigate whether irradiation-induced mitochondrial dysfunction contributes to the increase in the production/accumulation of ROS in mitochondria, we measured the levels of ROS in cells with the MitoSOX Red mitochondrial superoxide indicator as a specific probe of mitochondrial $\mathrm{O}_{2}^{-}$. Compared with that of the non-irradiated cells, the fluorescence intensity of irradiated cells had increased slightly after 18 hours and had increased more significantly at 24 hours after irradiation (Figure 4). More importantly, the increase of mitochondrial ROS levels continued up to 72 hours after irradiation, the longest time point included in this study.

\section{Oxidation of mitochondrial DNA after irradiation}

We wondered whether the increased ROS levels would induce oxidative damage of DNA. By measuring the levels of 8-OHdG, a biomarker of oxidative DNA damage, we found that the levels of 8-OHdG in mitochondrial DNA did not change significantly within 18 hours but increased significantly at times of 24 hours or later after irradiation (Figure 5). In contrast, no significant change in the 8-OHdG level in the nuclear DNA was observed within 72 hours after irradiation (Figure 5), but measurements at longer time points are needed.

\section{Discussion}

In this study, we found that gamma-ray radiation induced an instantaneous increase in the levels of intracellular ROS within minutes, a mitochondrial dysfunction within hours, and a persistent increase of mitochondrial ROS levels and oxidation of mitochondrial DNA within days. The clear pattern of the time courses associated with these changes help us to understand 
the relationships among mitochondrial dysfunction, oxidative stress, and radiation-induced genomic instability.

First, after gamma-ray irradiation, the intracellular ROS levels as detected by DCF fluorescence increased robustly within minutes and then disappeared very quickly (within 30 minutes). Because ionizing radiation can ionize $\mathrm{H}_{2} \mathrm{O}$ into ${ }^{\circ} \mathrm{OH}$, a toxic form of $\mathrm{ROS}$ with a life span as short as $10^{-9}$ seconds $[8,13]$, we thought that the instantaneous increase in intracellular ROS levels soon after irradiation could simply be the result of radiation-induced ionization of $\mathrm{H}_{2} \mathrm{O}$ into ${ }^{\circ} \mathrm{OH}$ and their byproducts.

Second, by measuring the activity of NADH dehydrogenase, mitochondrial dysfunction was identified within 12 hours after irradiation. Although the ${ }^{\circ} \mathrm{OH}$ radical has a very short life span, the transient but robust release of ${ }^{\circ} \mathrm{OH}$ after irradiation may oxidize biomolecules such as proteins or lipids in the cells. Cardiolipin, phosphatidylcholine, and phosphatidylethanolamine are composed of polyunsaturated fatty acids, which are especially susceptible to peroxidation by ROS $[15,16]$. These phospholipids are located in the inner mitochondrial membrane and are required for the optimum activity of ETC complexes, including complexes I and III $[34,35]$. Therefore, it was not surprising that the robust release of ${ }^{\circ} \mathrm{OH}$ soon after irradiation might damage the mitochondria within hours via the peroxidation of those phospholipids. However, it is unclear yet what oxidative injury occurs to each protein that constitutes these ETC complexes. A proteomic analysis, as well as the information on the oxidation state of the mitochondrial lipids may help to prove the hypothesis.

The enzyme complexes of the ETC are known to play a central role in the synthesis of ATP. During this process, $\mathrm{O}_{2}{ }^{-}$is generated due to the leakage of electrons from complex I or III [36]. If oxidative alterations to the phospholipid profile are induced by ${ }^{\circ} \mathrm{OH}$ as a result of radiation exposure, enhancement of $\mathrm{O}_{2}{ }^{-}$generation may arise from the leakage of electrons that is associated with the dysfunction of the ETC. A decreased activity of mitochondrial ETC complexes has recently been found to result in an increase in the cellular $\mathrm{H}_{2} \mathrm{O}_{2}$ level [37]. 
Because the appearance of the mitochondrial ROS was observed at a time of 24 hours after irradiation, which is a few hours later than the occurrence of mitochondrial dysfunction, we thought that the significant increase in ROS levels in the mitochondria could be caused by an increased leak of electrons from the mitochondrial ETC complexes. However, the decreased activity of manganese superoxide dismutase and some other enzymes might also accelerate the accumulation of ROS in the mitochondria [38].

Finally, we found that irradiation induced the oxidation of mitochondrial DNA at a time of 24 hours after irradiation, which is the same time that the ROS levels begin to significantly increase in mitochondria. It is known that the oxidation-reduction potential of deoxyguanosine is the lowest among the four types of nucleic acid bases of DNA, and that deoxyguanosine is susceptible to oxidative modification by ROS. Therefore, $8-\mathrm{OHdG}$, which is modified with a hydroxyl group on the $\mathrm{C} 8$ of guanine, is a highly sensitive biomarker of oxidative DNA damage [28]. However, the 8-OHdG in either mitochondrial DNA or nuclear DNA was not significantly changed by 18 hours after irradiation. This result suggests that the intracellular ROS, consisting primarily of ${ }^{\circ} \mathrm{OH}$ and generated immediately after irradiation, has little or no direct oxidative influence on the nuclear and mitochondrial DNA. Therefore, the oxidative damage of the mitochondrial DNA was more likely induced by the local accumulation of ROS due to mitochondrial dysfunction. Although we did not detect an increase of 8-OHdG in nuclear DNA even after irradiation with 5Gy, DNA deletion might be detectable by other assessments for DNA deletion, such as Comet assay and the formation of $\gamma$-H2AX foci in the nuclei.

In this study, the data regarding mitochondrial dysfunction and ROS levels in mitochondria were limited to measurements up to 72 hours after irradiation. The mitochondrial genome encodes 2 different types of mitochondrial rRNAs, 22 types of tRNAs, and 13 types of proteins that are components of the complexes of the ETC [39]. It has previously been demonstrated that adenine misincorporation occurs opposite to an 8-OHdG lesion during the replication of DNA and induces a G:C to T:A transversion [40]. If this transversion is induced 
by the accumulation of $8-\mathrm{OHdG}$, point mutations of the mitochondrial genome may evoke a permanent functional abnormality in the mitochondria. In fact, we have very recently observed that the generation of $\mathrm{O}_{2}^{-}$by mitochondria persists for over a week after irradiation (data not shown). However, further studies with measurements at longer time points are required to understand whether and how the persistent generation of ROS by mitochondria contributes to radiation-related genomic instability.

In summary, we observed an instantaneous and robust release of ${ }^{\circ} \mathrm{OH}$ soon after exposure of cells to ionizing radiation. The robust release of ${ }^{\circ} \mathrm{OH}$ oxidized the ETC complexes to induce mitochondrial dysfunction, which further caused persistent oxidative stress and induced the oxidation of mitochondrial DNA. Although we have not examined the genomic instability of cells in this study, we speculate that the mitochondrial dysfunction causes persistent oxidative stress and may then contribute to promote genomic instability for a long time after irradiation. Therefore, normalizing the mitochondrial function by the administration of antioxidants may be a potential therapeutic strategy for radiation-induced genomic instability and carcinogenesis.

\section{Acknowledgements}

This work was supported in part by a Grant-in-Aid for the Global Centers of Excellence Program from the Ministry of Education, Science, Sports, Culture and Technology of Japan.

\section{Declaration of interest}

The authors confirm no conflicts of interest. The authors alone are responsible for the content and writing of the paper. 


\section{References}

[1] Rothkamm K, Kühne M, Jeggo PA, Löbrich M. Radiation-induced genomic rearrangements formed by nonhomologous end-joining of DNA double-strand break. Cancer Res 2001;61:3886-3893.

[2] Huang L, Snyder AR, Morgan WF. Radiation-induced genomic instability and its implications for radiation carcinogenesis. Oncogene 2003;22:5848-5854.

[3] Roy K, Kodama S, Suzuki K, Fukase K, Watanabe M. Hypoxia relieves X-ray-induced delayed effects in normal human cells. Radiat Res 2000; 154:659-666.

[4] Leach JK, Van Tuyle G, Lin PS, Schmidt-Ullrich R, Mikkelsen RB. Ionizing radiation-induced, mitochondria-dependent generation of reactive oxygen/nitrogen, Cancer Res 2001;61:3894-3901.

[5] Tominaga H, Kodama S, Matsuda N, Suzuki K, Watanabe M. Involvement of reactive oxygen species (ROS) in the induction of genetic instability by radiation. J Radiat Res 2004;45:181-188.

[6] Kim GJ, Fiskum GM, Morgan WF. A role for mitochondrial dysfunction in perpetuating radiation-induced genomic instability. Cancer Res 2006;66:10377-10383.

[7] Motoori S, Majima HJ, Ebara M, Kato H, Hirai F, Kakinuma S, Yamaguchi C, Ozawa T, Nagano T, Saisho H. Overexpression of mitochondrial manganese superoxide dismutase protects against radiation-induced cell death in the human hepatocellular carcinoma cell line HLE. Cancer Res 2001;61:5382-5388.

[8] Robbins MEC, Zhao W. Chronic oxidative stress and radiation-induced late normal tissue injury. Int J Radiat Biol 2004;80:251-259.

[9] Turrens JF, Boveris A. Generation of superoxide anion by the NADH dehydrogenase of bovine heart mitochondria. Biochem J 1980;191:421-427.

[10] Demin OV, Kholodenko BN, Skulachev VP. A model of $\mathrm{O}_{2}{ }^{-}$in the complex III of the electron transport chain. Mol Cell Biochem 1998;184:21-33.

[11] Gille L, Nohl H. The ubiquinol/bc $c_{1}$ redox couple regulates mitochondrial oxygen radical formation. Arch Biochem Biophys 2001;388:34-38.

[12] Han D, Antunes F, Canali R, Rettori D, Cadenas E. Voltage-dependent anion channels control the release of the superoxide anion from mitochondria to cytosol. J Biol Chem 2003;278:5557-5563.

[13] Riley PA. Free radicals in biology: oxidative stress and the effects of ionizing radiation. Int 
J Radiat Biol 1994;65:27-33.

[14] Wallace SS. Enzymatic processing of radiation-induced free radical damage in DNA. Radiat Res 1998;150:S60-S79.

[15] Chicco AJ, Sparagna GC. Role of cardiolipin alterations in mitochondrial dysfunction and disease. Am J Physiol Cell Physiol 2007;292:33-44.

[16] Dekker CJ, Geurts van kessel WSM, Klomp JPG, Pieters J, De Kruijef B. Synthesis and polymorphic phase behaviour of polyunsaturated phosphatidylcholines and phosphatidylethanolamines. Chem Phys Lipids 1983;33:93-106.

[17] Urata Y, Goto S, Kawakatsu M, Yodoi J, Eto M, Akishita M, Kondo T. DHEA attenuates PDGF-induced phenotypic proliferation of vascular smooth muscle A7r5 cells through redox regulation. Biochem Biophys Res Commun 2010;396:489-494.

[18] Brandt R, Keston AS. Synthesis of diacetyldichlorofluorescin: A stable reagent for fluorometric analysis. Anal Biochem 1965;11:6-9.

[19] Keston AS, Brandt R. The fluorometric analysis of ultramicro quantities of hydrogen peroxide. Anal Biochem 1965; 11:1-5.

[20] Cathcart R, Schwiers E, Ames BN. Detection of picomole levels of hydroperoxides using a fluorescent dichlorofluorescein assay. Anal Biochem 1983;134:111-116.

[21] Jakubowski W, Bartosz G. 2,7-dichlorofluorescin oxidation and reactive oxygen species: What does it measure?. Cell Biol Int 2000;24:757-760.

[22] Choksi KB, Nuss JE, Boylston WH, Rabek JP, Papaconstantinou J. Age-related increases in oxidatively damaged proteins of mouse kidney mitochondrial electron transport chain complexes. Free Radic Biol Med 2007;43:1423-1438.

[23] Trounce IA, Kim YL, Jun AS, Wallace DC. Assessment of mitochondrial oxidative phosphorylation in patient muscle biopsies, lymphoblasts, and transmitochondrial cell lines. Methods Enzymol 1996;264:484-509.

[24] Kwong LK, Sohai RS. Age-related changes in activities of mitochondrial electron transport complexes in various tissues of the mouse. Arch Biochem Biophys 2000;373:16-22.

[25] Jarreta D, Orús J, Barrientos A, Miró O, Roig E, Heras M, Moreas CT, Cardellach F, Casademont J. Mitochondrial function in heart muscle from patients with idiopathic dilated cardiomyopathy. Cardiovasc Res 2000;45:860-865.

[26] Robinson KM, Janes MS, Pehar M, Monette JS, Ross MF, Hagen TM, Murphy MP, Bechman JS. Selective fluorescent imaging of superoxide in vivo using ethidium-based 
probes. Proc Natl Acad Sci USA 2006;103:15038-15043.

[27] Munusamy S, MacMillan-Crow LA. Mitochondrial superoxide plays a crucial role in the development of mitochondrial dysfunction during high glucose exposure in rat renal proximal tubular cells. Free Radic Biol Med 2009;46:1149-1157.

[28] Kasai H, Hayami H, Yamaizumi Z, Saito H, Nishimura S. Detection and identification of mutagens and carciogens as their adducts with guanosine derivatives. Nucleic Acids Res 1984; 12:2127-2136.

[29] Spitz DR, Azzam EI, Li JJ, Gius D. Metabolic oxidation/reduction reactions and cellular responses to ionizing radiation: A unifying concept in stress response biology. Cancer Metastasis Rev 2004;23:311-322.

[30] Esposti MD. Measuring mitochondrial reactive oxygen species. Methods 2002;26:335-340.

[31] Raha S, Robinson BH. Mitochondria, oxygen free radicals, disease and ageing. Trends Biochem Sci 2000;25:502-508.

[32] Turrens JF. Superoxide production by the mitochondrial respiratory chain. Biosci Rep 1997; 17:3-8.

[33] Solaini G, Harris DA. Biochemical dysfunction in heart mitochondria exposed to ischaemia and reperfusion. Biochem J 2005;390:377-394.

[34] Daum G. Lipids of mitochondria. Biochim Biophys Acta 1985;822:1-42 .

[35] Fry M, Green DE. Cardiolipin requirement for electron transfer in complex I and III of the mitochondrial respiratory chain. J Biol Chem. 1981;256:1874-1880.

[36] Murphy MP. How mitochondria produce reactive oxygen species. Biochem J 2009;417:1-13.

[37] She H, Yang Q, Shepherd K, Smith Y, Miller G, Testa C, Mao Z. Direct regulation of complex I by mitochondrial MEF2D is disrupted in a mouse model of Parkinson disease and in human patients. J Clin Invest 2011;121:930-940.

[38] Park J, Lee J, Choi C. Mitochondrial network determines intracellular ROS dynamics and sensitivity to oxidative stress through switching inter-mitochondrial messengers. PLoS ONE 2011;6:e23211.

[39] Boore JL. Animal mitochondrial genomes. Nucleic Acids Res 1999;27:1767-1780.

[40] Shibutani S, Takeshita M, Grollman AP. Insertion of specific bases during DNA synthesis past the oxidation-damaged base 8-oxodG. Nature 1991;349:431-434. 
Figure Legends

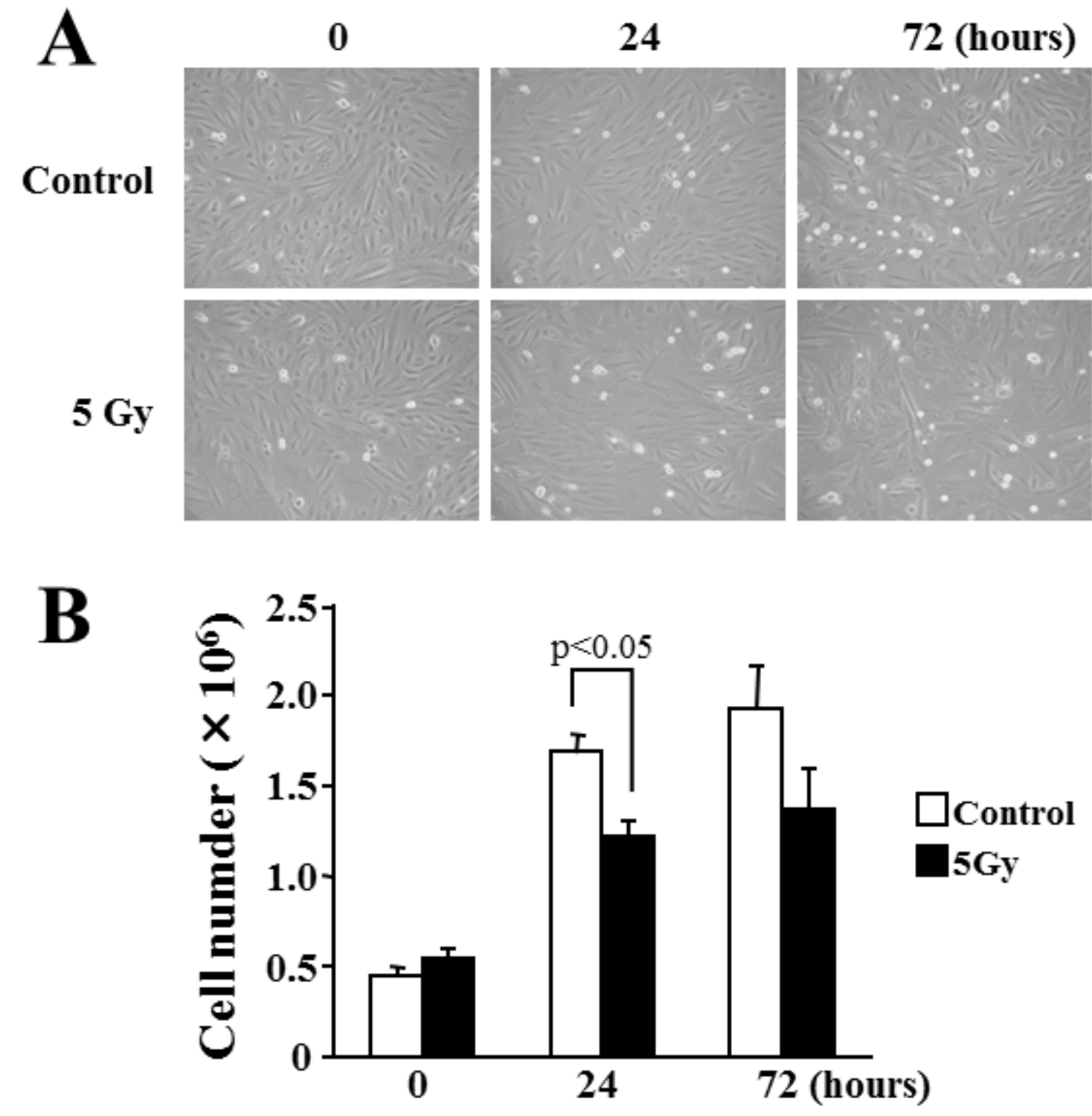

Figure 1. Cell growth after irradiation. The morphological changes of cells were recorded by digital camera under microscopy. The numbers of survived cells were counted by a cell counter. Although the morphological findings in cells survived from radiation did not obviously change (A), the decreased number of cells indicated an inhibition of cell growth 24 and 72 hours after 5 Gy irradiation (B). 


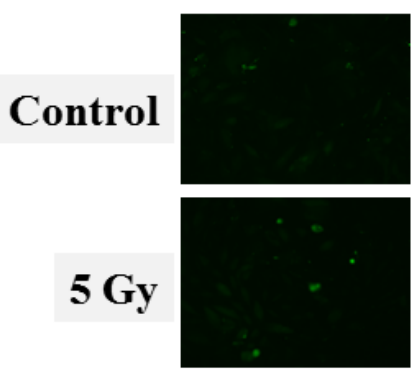

$\mathbf{0}$
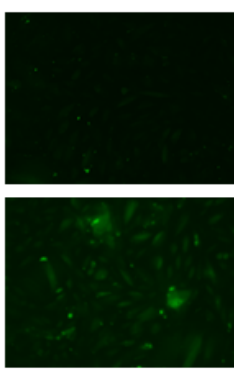

5
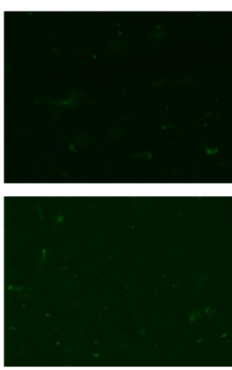

30
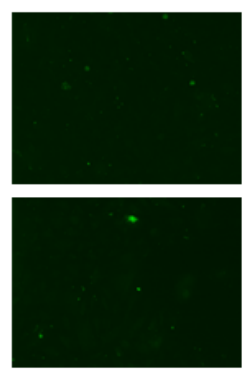

60
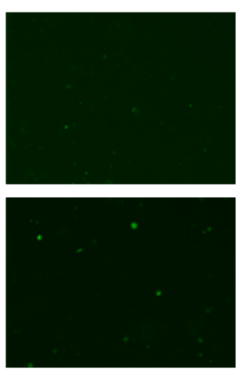

120 (minutes)

Figure 2. Time course changes in the intracellular ROS levels after irradiation. Cells were incubated with $\mathrm{H}_{2}$ DCFDA for 20 minutes before irradiation, and intracellular ROS levels were detected by DCF fluorescence (green) at 5, 30, 60, or 120 minutes after irradiation. The DCF fluorescence showed an obvious increase at 5 minutes after irradiation (lower panel). However, the intracellular ROS levels were barely detected at 30,60, and 120 minutes after irradiation, which was comparable with the ROS levels observed for non-irradiated cells (upper panel). 


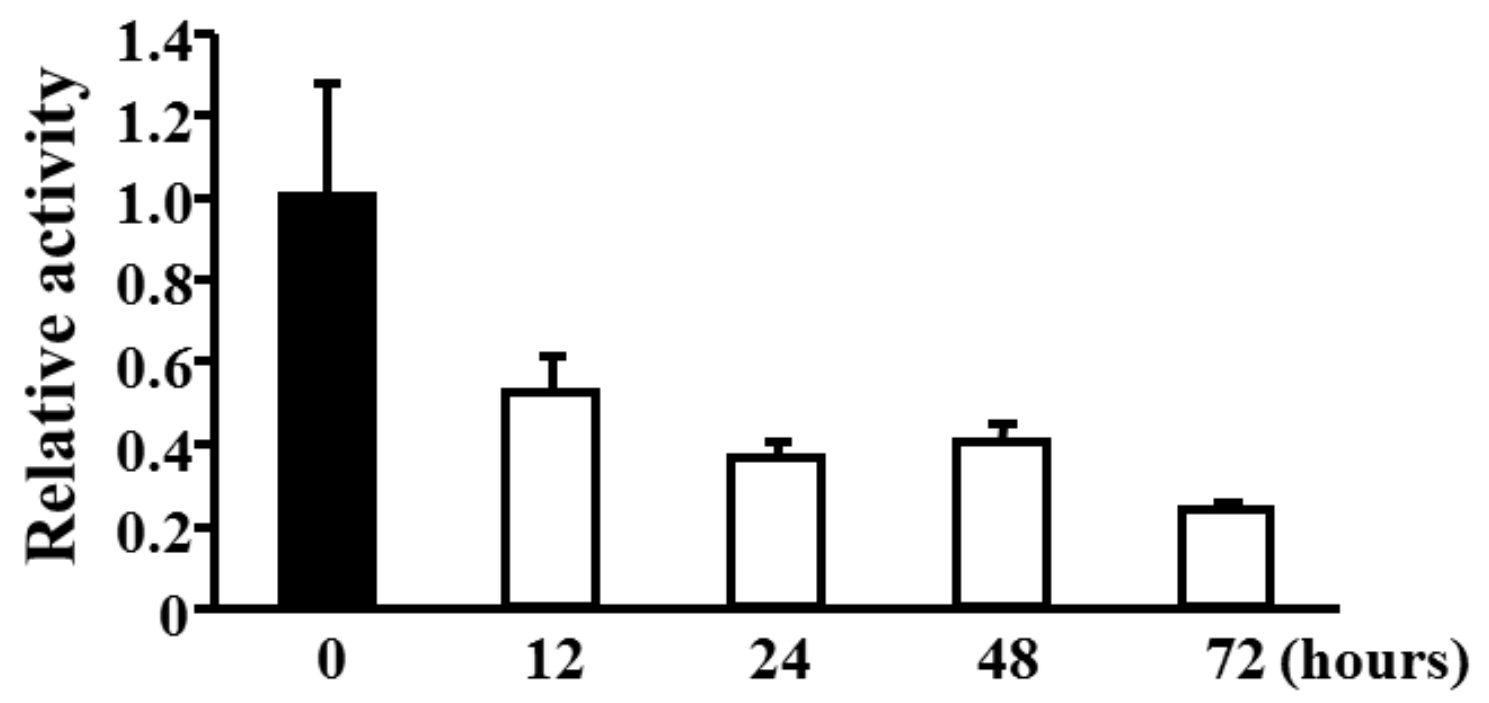

Figure 3. Time course changes of the activity of NADH dehydrogenase after irradiation. The mitochondrial dysfunction was evaluated by the activity of NADH dehydrogenase. The activity of NADH dehydrogenase decreased to approximately $50 \%$ of baseline at 12 hours and remained at this lower level until 72 hours after irradiation. Each value is given as the activity relative to the control group at each time point, and data are represented as the mean \pm SEM of three independent experiments. 

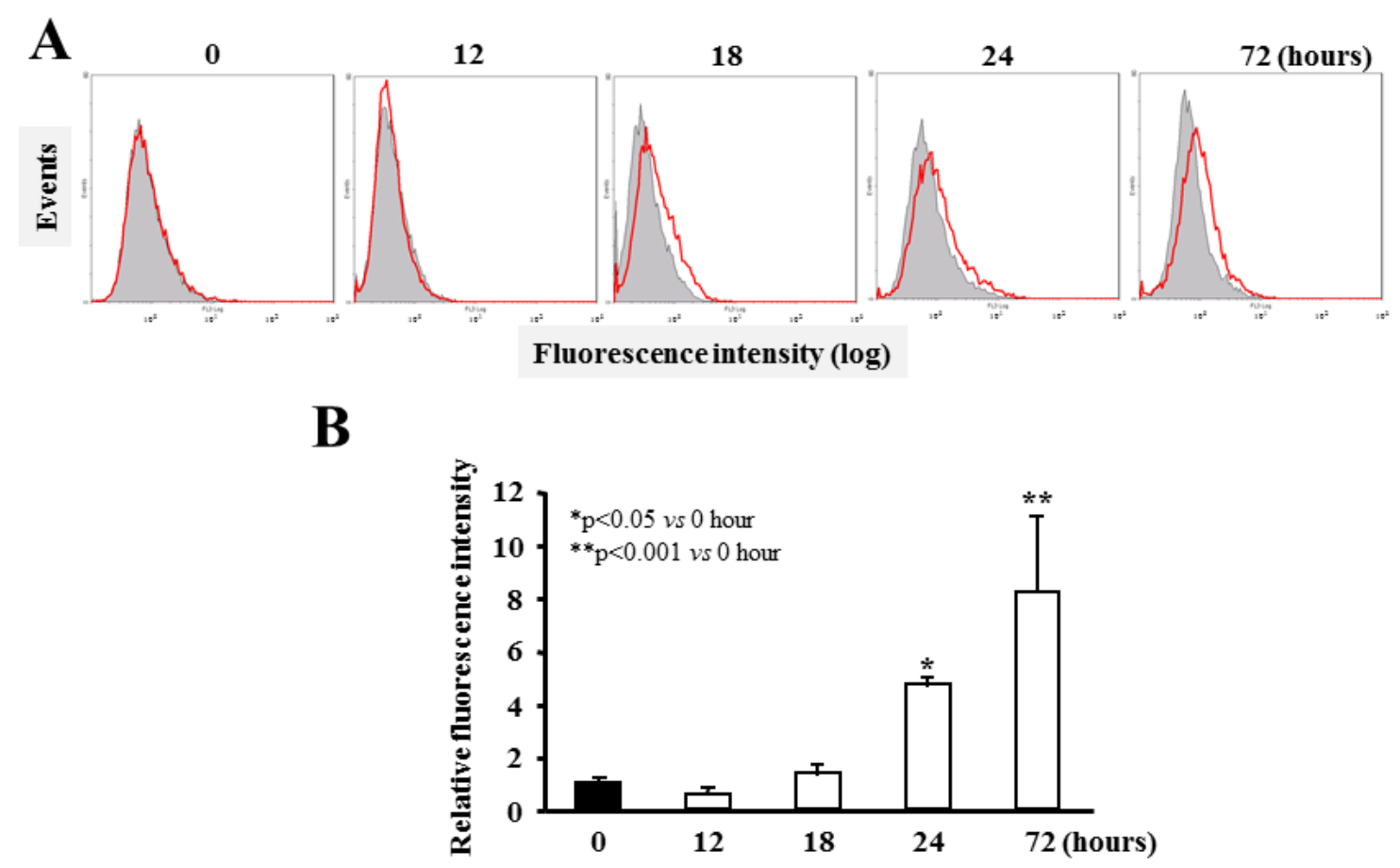

Figure 4. Time course changes in ROS levels in mitochondria after irradiation. ROS levels in the mitochondria of cells after irradiation were analyzed by flow cytometry with a MitoSOX Red mitochondrial superoxide indicator. (A) Representative histograms at each time point are shown, and the gray filled histogram and red line histogram indicate the results of non-irradiated cells and irradiated cells, respectively. (B) A semi-quantitative analysis showed that the relative ratio of the mean fluorescence intensity increased significantly in cells 24 and 72 hours after radiation. Each value represents the mean \pm SEM of 3 to 5 separate experiments. 


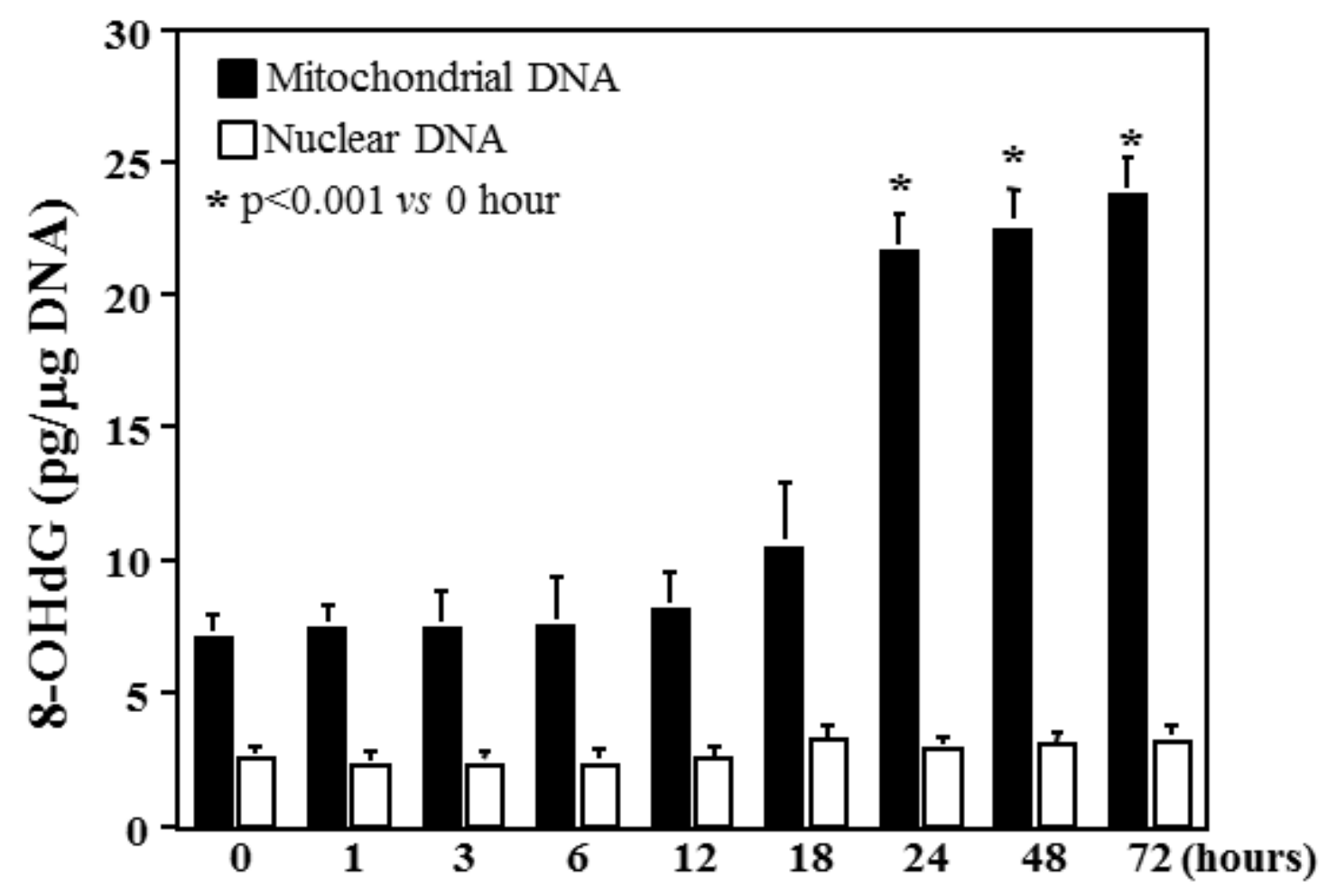

Figure 5. DNA oxidation at different time points after irradiation. Nuclear and mitochondrial DNA was purified by using the DNA Extractor TIS Kit and the mtDNA Extractor CT Kit, respectively. DNA oxidation was evaluated by measuring the levels of 8-OHdG using ELISA kit. The levels of 8-OHdG in the mitochondrial DNA significantly increased at 24 hours or later after irradiation, but no significant changes in the nuclear DNA was observed within 72 hours. Each value represents the mean $\pm \mathrm{SEM}$ of three independent experiments. 